Fisioter Bras 2021;22(4);609-24

doi: $10.33233 / \mathrm{fb} . v 22 \mathrm{i} 4.4527$

\title{
REVISÃO
}

\section{Cinesioterapia aplicada à fadiga oncológica}

Kinesiotherapy applied to oncological fatigue

Raynara Kéllen Pinto Moreira*, Barbara Kelly de Souza Barreto*, Lucicleia Nascimento Santos*, Yandra Alves Prestes*, Maria Gabriela de Andrade Lucena, Ft.**, Hércules Lázaro Morais Campos, Ft., M.Sc. ${ }^{* *}$

*Graduanda de Fisioterapia da Universidade Federal do Amazonas (UFAM), Instituto de Saúde e Biotecnologia (ISB), Coari, AM, ${ }^{* *}$ Fisioterapeuta do Hospital Paulistano A. C. Camargo, Pós-Graduada em Fisioterapia Oncológica e Hospitalar pelo Hospital A. C. Camargo de São Paulo, Título de Especialista em Oncologia pela ABFO/COFFITO e Mestranda em Ciência Cirúrgica pelo Departamento de Cirurgia Vascular pela Universidade Federal de São Paulo (UNIFESP), ${ }^{* *}$ Doutorando em Saúde Coletiva, Docente de Fisioterapia da Universidade Federal do Amazonas (UFAM), Instituto de Saúde e Biotecnologia (ISB), Coari, AM

Recebido em 22 de dezembro de 2020; Aceito em 13 de agosto de 2021.

Correspondência: Raynara Kéllen Pinto Moreira, Beco dos Amorins, 137, Chagas Aguiar, 69460-000 Coari AM, E-mail: raynara.moreira16@gmail.com

Raynara Kéllen Pinto Moreira: raynara.moreira16@gmail.com Barbara Kelly de Souza Barreto: barbara.sbarreto@hotmail.com Lucicleia Nascimento Santos: nascimento.santos.lucicleia@gmail.com Yandra Alves Prestes: yprestess18@hotmail.com Maria Gabriela de Andrade Lucena: gabrielalucena19@yahoo.com.br Hércules Lázaro Morais Campos: herculesImc@hotmail.com

\section{Resumo}

Objetivo: Buscou-se na literatura estudos que façam uso da cinesioterapia para tratar, atenuar e melhorar a fadiga em pacientes oncológicos além de identificar qual melhor intervenção para prática clínica com esse grupo de pacientes. Métodos: Realizou-se uma revisão integrativa da literatura acompanhada com avaliação de qualidade metodológica. Iniciaram-se as buscas no período de 7 de maio de 2020 a 15 de setembro de 2020, nas bases de dados PEDro, Pubmed e Scielo. Foram encontrados 
146 artigos, porém apenas 12 foram selecionados e analisados de acordo com os critérios de inclusão e a qualidade metodológica da escala PEDro. Resultados: Os estudos encontrados apresentam diversas condutas fisioterapêuticas para a fadiga em pacientes com idade entre 18 e 94 anos de curto a longo prazo, sendo no mínimo 3 meses e no máximo 2 anos de intervenção em níveis ambulatoriais e domiciliares, com protocolos de exercícios terapêuticos aeróbicos, ativos, resistidos e funcionais que proporcionaram resultados positivos aos pacientes com fadiga oncológica. Conclusão: O uso da fisioterapia com exercícios cinesioterapêuticos é essencial para reabilitação desses pacientes, fazendo-se necessário mais pesquisas com ensaios clínicos incluindo a aplicação da fisioterapia com exercícios cinesioterapêuticos.

Palavras-chave: oncologia; fisioterapia; fadiga.

\section{Abstract}

Objective: We sought in the literature studies that use kinesiotherapy to treat, mitigate and improve fatigue in cancer patients, in addition to identifying which is the best intervention for clinical practice with this group of patients. Methods: An integrative literature review was carried out, accompanied by methodological quality assessment. Searches started in the period from May 7, 2020 to September 15, 2020, in the PEDro, Pubmed and Scielo databases. 146 articles were found, however, only 12 were selected and analyzed according to the inclusion criteria and methodological quality of the PEDro scale. Results: The studies found several physiotherapeutic approaches to fatigue in patients aged 18 to 94 years in the short to long term, with a minimum of 3 months and a maximum of 2 years of intervention at outpatient and home levels, with protocols for aerobic therapeutic exercises, active, resisted and functional that provided positive results to patients with cancer fatigue. Conclusion: The use of physical therapy with kinesiotherapeutic exercises is essential for the rehabilitation of these patients, making necessary further research with clinical trials.

Keywords: oncology; physical therapy; fatigue.

\section{Introdução}

A fadiga relacionada ao câncer (FRC) consiste em uma sensação de cansaço extremo [1], sendo classificada pelo Consenso Brasileiro de Fadiga em dimensões físicas, cognitivas e afetivas. Na dimensão física ocorre uma redução de energia, na cognitiva há diminuição da atenção, da concentração e da memória e na afetiva apresenta uma baixa motivação ou desinteresse [1-4]. Além disso, ela pode ser 
classificada em primária e secundária. A fadiga primária é consequência da própria doença, a secundária é resultante de síndromes concomitantes, comorbidades $[2,5,6]$.

Mundialmente a fadiga é um dos sintomas que mais afetam pacientes oncológicos, interferindo na sua qualidade de vida (QV) e Capacidade Funcional (CF) e é desencadeada por fatores pós-quimioterapia e radioterapia [7-10]. Sua incidência em estudos científicos varia entre $15-80 \%$ dos pacientes [11]. Estima-se que $50 \%$ a $90 \%$ dos pacientes com câncer apresentam fadiga [7-10], que essa sintomatologia pode persistir por meses e até anos após o tratamento quimioterápico ou radioterápico [8-12].

A cinesioterapia é o recurso que o fisioterapeuta usa para tratar diversas disfunções nos pacientes de modo geral, consiste em exercícios terapêuticos, que utilizam de movimentos do corpo como forma de intervenção para reduzir os sintomas, melhorar e manter a função e a retomada das atividades de vida diária [13].

Pretende-se, então, buscar na literatura estudos que façam uso da cinesioterapia para tratar, atenuar e melhorar a fadiga em pacientes oncológicos.

\section{Métodos}

Estratégia de pesquisa e seleção dos estudos

Realizou-se uma revisão integrativa da literatura acompanhada com avaliação de qualidade metodológica. Iniciaram-se as buscas no período de 07 de maio de 2020 até 15 de setembro de 2020. A pesquisa foi realizada nas bases de dados PEDro, Pubmed e Scielo. Utilizaram-se os termos de busca nos três idiomas, português e inglês: "oncological fatigue", "physiotherapy + oncological fatigue", "exercise + oncological fatigue", "kinesiotherapy + oncological fatigue", "treatment + oncological fatigue", "kinesiotherapy + fatigue + cancer". A identificação e seleção dos estudos foram de acordo com os títulos, palavras-chave e resumos dos estudos. Notou-se que muitos outros não se caracterizavam com os critérios de inclusão para avaliação, formalizando assim uma nova revisão de texto completa para a elegibilidade usando os critérios de inclusão/exclusão (fluxograma 1).

\section{Avaliação metodológica dos estudos}

Os estudos que cumpriram os critérios de inclusão e não constavam nota foram avaliados por mais de um examinador de forma independente quanto à qualidade metodológica da escala PEDro, a mais usada na área da reabilitação. Essa escala de qualidade metodológica foi desenvolvida pela Physiotherapy Evidence Database 
constituindo uma pontuação total de até 10 pontos, incluindo 11 critérios de avaliação. Sendo assim, nenhum estudo foi excluído pela baixa qualidade metodológica (escore PEDro menor que 5), correspondendo aos devidos critérios de inclusão para o referente estudo. Os detalhes metodológicos do estudo estão descritos no fluxograma abaixo:

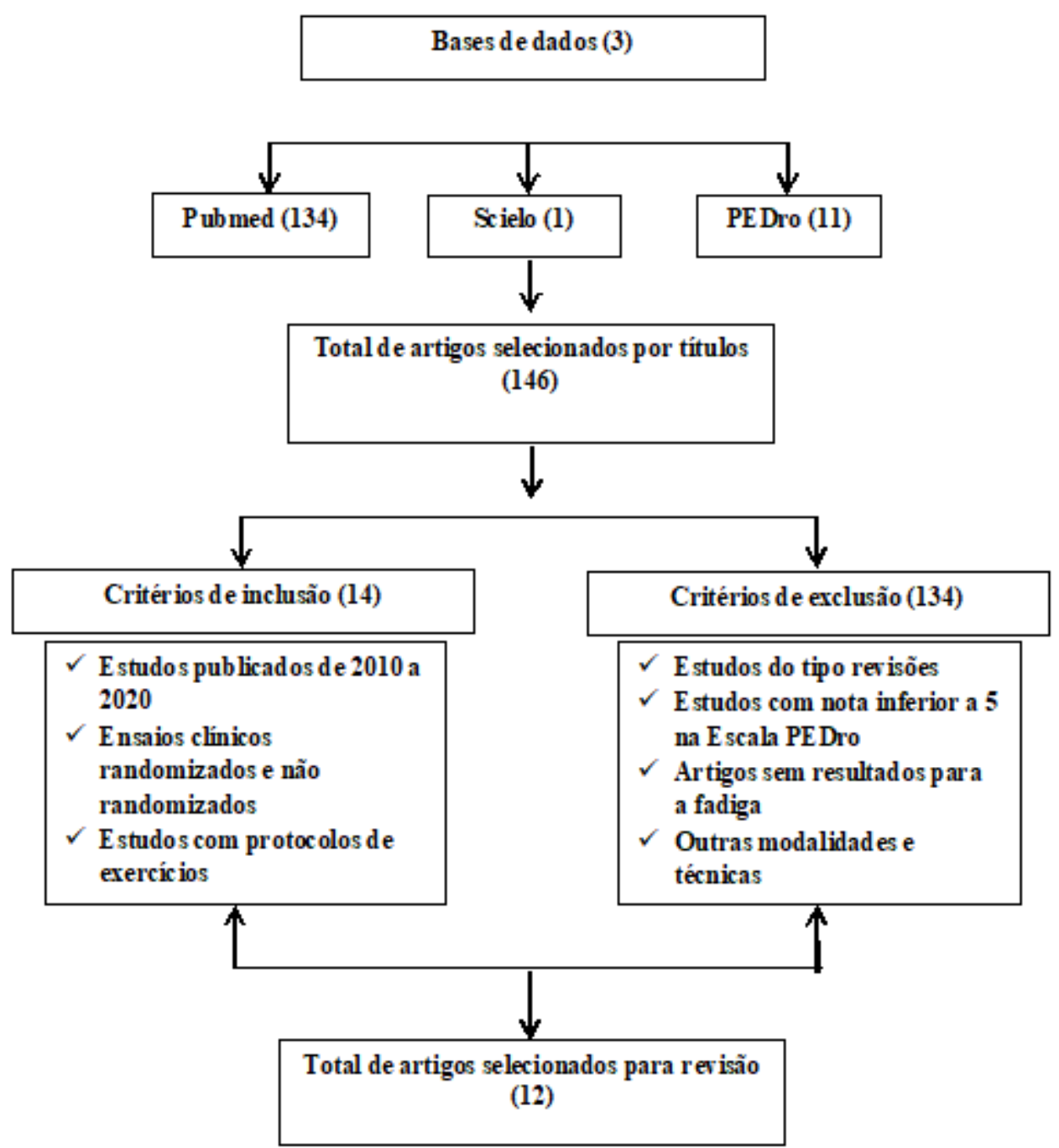

Fluxograma 1 - Estratégia de seleção de estudos

\section{Resultados}

Com base nas análises das evidências científicas constatou-se que a média de idade dos pacientes encontrados variou de 18 a 94 anos. Em geral, os estudos apontam que a presença de fadiga relacionada ao câncer (FRC) com uma incidência 15-80\% nos pacientes em tratamento oncológico [11], e uma prevalência de 50\% a 90\% nesse mesmo grupo $[7,8]$. 
A conduta fisioterapêutica para tratamento dessa fadiga está descrita na literatura de curto a longo prazo, sendo no mínimo 3 meses e no máximo 2 anos de intervenção em níveis ambulatoriais e domiciliares. Encontraram-se protocolos de exercícios terapêuticos aeróbicos, ativos, resistidos e funcionais que proporcionaram resultados positivos aos pacientes com fadiga oncológica. Outras informações estão abaixo no quadro 1.

Quadro 1 - Protocolos dos exercícios terapêuticos para pacientes com fadiga oncológica (ver PDF)

\section{Discussão}

Os estudos encontrados apresentam intervenções para fadiga relacionada ao câncer (FRC) que são constituídos por programas de fisioterapia, que inclui exercícios ativos, treino de força, exercícios aeróbicos, exercícios de resistência e exercícios com carga, que trazem resultados positivos de curto a longo prazo para esses pacientes [2132].

Pyszora et al. [21] buscaram componentes para o programa de tratamento que consistiu em uma intervenção adaptada às condições dos pacientes participantes, tais como, exercícios ativos combinados com facilitação neuromuscular proprioceptiva (FNP) e técnicas liberação miofascial com estimulação respiratória que proporcionam melhoras no bem-estar geral do paciente, pois tais técnicas não requerem grandes gastos de energia dos pacientes em relação aos métodos que os envolvem ativamente, sendo assim apresentam redução na fadiga, além de diminuir a sintomatologia coexistentes, como dor, sonolência, falta de apetite e depressão e melhoraram a força muscular dos pacientes e sua aptidão geral [21]. Baumann et al. [25] concluíram que um programa de exercícios adaptado as preferências, características e recursos físicos de cada indivíduo levou a diminuição dos efeitos colaterais do tratamento ao longo do tempo, do que apenas os cuidados habituais [25]. Sendo também justificado esse fator no estudo de Rogers et al. [31] que desenvolveram um programa que consiste em exercícios aeróbicos, de intensidade moderada e treinamento resistido, que no decorrer do treinamento os exercícios foram intensificando-se significativamente, mostrando que a intensidade dos exercícios adaptada ao estágio contribui para diminuir a percepção da fadiga, deixando o paciente mais disposto a executar as atividades e encarar 0 tratamento contra o câncer [31].

De acordo com Schmidt et al. [26], o início precoce do exercício pode prevenir o ciclo vicioso de força muscular prejudicada, baixa atividade física, fadiga e maior redução da atividade física, o que pode levar a problemas de saúde a longo prazo, 
mesmo após a conclusão bem-sucedida do tratamento do câncer. Concluíram que treinamento de resistência progressiva supervisionado, deve ser oferecido como parte integrante dos cuidados de suporte para pacientes com câncer em quimioterapia.

Desta forma Husebø et al. [22] utilizaram-se de treinamento de força com faixas de resistência para braços e pernas e treinamento de força para a parte superior do corpo, em mulheres com fadiga relacionada ao câncer, durante todo período de quimioterapia adjuvante. Segundo Samuel et al. [23], os exercícios podem ser incorporados como parte da rotina do atendimento ao paciente durante o curso da CRT em pacientes com CCP [23].

Ammitzbøll et al. [30] aplicaram exercícios de resistência progressiva em pacientes após a cirurgia de câncer de mama com um grupo de sintomas, incluindo dor, insônia e fadiga, e a intervenção melhorou significativamente o estado de saúde e funcional. Desta forma, Grote et al. [27] abordaram exercícios de resistência, durante a radioterapia, defendem exercícios com carga, que mostra resultados ligeiramente melhores para a fadiga geral em pacientes com câncer de cabeça e pescoço [27].

Kampshoff et al. [32] concluíram em seu estudo que exercícios de intensidade alta e resistência apresentam melhora na fadiga geral em pacientes com câncer, com acompanhamento a longo prazo [32]. De acordo com Taaffe et al. [24], os efeitos de longo prazo dos exercícios e o impacto potencial de diferentes modalidades de exercícios (resistência + carga de impacto, aeróbio + resistência, aeróbio apenas) sobre a fadiga em pacientes com câncer de próstata submetidos a ADT tiveram um efeito benéfico na fadiga e vitalidade, e para pacientes com os níveis mais altos de fadiga e vitalidade mais baixa melhoraram com exercícios [24].

Van Weert et al. [28] abordaram treinamento físico combinado com terapia cognitivo-comportamental e apenas treinamento físico. De modo geral, os exercícios de treino muscular de resistência progressiva apresentaram como resultado efeitos significativos e benéficos sobre a fadiga. Andhare et al. [29] sugerem que a combinação de treinamento aeróbico domiciliar e caminhada, que enfoca o efeito da caminhada aeróbica programada versus caminhada individual sobre a fadiga e a qualidade de vida no câncer de pacientes após 4 semanas de treinamento, apresentou efeito benéfico [29].

Portanto, é imprescindível o uso de exercícios terapêuticos para fadiga desencadeada por tratamento oncológico. Estudos mostram uma eficaz redução da fadiga, melhora da qualidade de vida e capacidade funcional [8,14-15], além disso, pode-se ressaltar que um dos fatores que alteram a qualidade de vida desses pacientes está associado a diminuição da massa muscular, devido à sarcopenia, o que afeta a sua qualidade de vida, uma vez que o paciente passa a ter uma fraqueza muscular e maior fadiga [18-20]. Desta forma, o exercício físico regularmente pode aumentar a 
capacidade funcional, reduzindo assim a energia necessária para suportar as atividades diárias, melhorando a qualidade de vida desse paciente $[8,16,17]$. Pode-se concluir que a intervenção aplicada a fadiga oncológica que Pyszora et al. [21] realizaram, tornou-se adaptável ao paciente, reduzindo fadiga e outras sintomatologias. Schmidt et al. [26] abordam o início precoce do exercício para prevenir o ciclo vicioso de força muscular.

\section{Conclusão}

Há uma lacuna quanto às publicações de ensaios clínicos da fisioterapia que utilizam a cinesioterapia aplicada a fadiga oncológica, tanto em níveis ambulatoriais quanto domiciliares.

Exercícios aeróbicos, ativos, resistidos e funcionais aplicados a pacientes com fadiga oncológica devem ser de curto a longo prazo, sendo no mínimo de 3 semanas a 2 anos, com 10 a 30 minutos, de 3 a 5 dias por semana, com repetições de 8 a 12 e series de 3 , que proporcionam melhoras nos níveis de fadiga, além de reduzir sintomatologias coexistentes, melhorando qualidade de vida e capacidade funcional desses pacientes.

\section{Referências}

1. Instituto Nacional de Câncer. Cuidados Paliativos Oncológicos - Controle de Sintomas. Rev Bras Cancerol [Internet]. 2002 [cited 2021 Aug 13];48(2):191-1. Available from: https://rbc.inca.gov.br/revista/index.php/revista/article/view/2245Psicomotora

2. Oliveira SMC, Santos EV, Silva YMGPA. Cuidados paliativos: prevalência de fadiga em pacientes pediátricos. Rev Soc Bras Clin Med [Internet]. 2017 [cited 2021 Aug 13];15(4):240-5. Available from: https://docs.bvsalud.org/biblioref/2018/01/877081/154240-245.pdf

3. Mota DD, Cruz DA, Pimenta CA. Fadiga: uma análise do conceito. Acta Paul Enferm 2005;3(18):285-93. doi: 10.1590/S0103-21002005000300009

4. Mota DD, Pimenta CA. Fadiga em pacientes com câncer avançado: conceito, avaliação e intervenção. Rev Bras Cancerol 2002;48(2). doi: 10.32635/21769745.RBC.2002v48n4.2172

5. Chaudhuri A, Behan PO. Fatigue in neurological disorders. Lancet 2004;363(9413):97888. doi: 10.1016/S0140-6736(04)15794-2

6. Amaducci CM, Mota DD, Pimenta CA. Fadiga entre estudantes de graduação em Enfermagem. Rev Esc Enferm USP 2010;44(4):1052-8. doi: 10.1590/s008062342010000400028

7. Mendes LC, Barichello E. Intervenções no manejo da fadiga e qualidade de vida em pacientes em quimioterapia: estudo de revisão. Cogitare Enferm 2019(24). doi: /10.5380/ce.v24i0.61790 
8. Campos MPO, Hassan BJ, Riechelmann R, Giglio A. Fadiga relacionada ao câncer: uma revisão. Rev Assoc Med Bras 2011;57(2):211-9. doi: 10.1590/S010442302011000200021

9. Curt GA, Breitbart W, Cella D, Groopman JE, Horning Sj, Itri LM, et al. Impact of cancer-related fatigue on the lives of patients: new findings from the fatigue coalition. Oncologist 2000;5(5):353-60. doi: 10.1634/theoncologist.5-5-353

10. Wang XS, Giralt SA, Mendoza TR, Engstrom MC, Johnson BA Peterson N, et al. Clinical factors associated with cancer-related fatigue in patients being treated for leukemia and non-Hodgkin's lymphoma. J Clin Oncol 2002;20(5):1319-28. doi: 10.1200/JCO.2002.20.5.1319

11. Lawrence DP, Kupelnick BM, Miller K, Devine D, Lau J. Evidence report on the occurrence, assessment, and treatment of fatigue in cancer patients. J Natl Cancer Inst Monogr 2004;(32):40-50. doi: 10.1093/jncimonographs/lgh027

12. Cella D, Davis K, Breitbart W, Curt G. Cancer related fatigue: prevalence of proposed diagnostic criteria in a United States sample of cancer survivors. J Clin Oncol 2001;19(14):3385-91. doi: 10.1200/jco.2001.19.14.3385

13. Kisner C, Colby LA. Exercícios terapêuticos: fundamentos e técnicas. $6^{\underline{a}}$ ed. Barueri: Manole; 2016.

14. Velthuis MJ, Agasi-Idenburg SC, Aufdemkampe G, Wittink HM. The effect of physical exercise on cancer-related fatigue during cancer treatment: a meta-analysis of randomised controlled trials. Clin Oncol 2010;22(3):208-21. doi: 10.1016/j.clon.2009.12.005

15. Vallance JKH, Couneya KS, Plotnikoff RC, Yasui Y, Mackey JR. Randomized controlled trial of the effects of print materials and step pedometers on physical activity and quality of life in breast cancer survivors. J Clin Oncol 2007;25(17):2352-9. doi: 10.1200/JCO.2006.07.9988

16. Lucía $A$, Earnest $C$, Pérez M. Cancer-related fatigue: can exercise physiology assist oncologists? Lancet Oncol 2003;4(10):616-25. doi: 10.1016/s1470-2045(03)01221-x

17. Rodrigues LF. A redução da fadiga oncológica através do exercício físico. Rev Bras Fisiol Exerc 2019;18(1):51-7. doi: 10.33233/rbfe.v18i1.2879

18. Duarte ECPS, Sousa RR, Feijó-Figueiredo MC, Pereira-Freire JA. Assistência nutricional para os cuidados paliativos de pacientes oncológicos: uma revisão integrativa. Revista de Atenção à Saúde 2020;18(64):124-32. doi: 10.13037/ras.vol18n64.6585

19. Frio CC, Pretto ADB, Gonzales MC, Pastore CA. Influência da composição corporal sobre a qualidade de vida de pacientes com câncer. Rev Bras Cancerol 2015;61(4):351-7. doi: 10.32635/2176-9745.RBC.2015v61n4.224

20. Vega MCMD, Laviano A, Pimentel GD. Sarcopenia e toxicidade mediada pela quimioterapia. Einstein (São Paulo) 2016;14(4):580-4. doi: 10.1590/S167945082016MD3740 
21. Pyszora A, Budzyński J, Wójcik A, Prokop A, Krajnik M. Physiotherapy programme reduces fatigue in patients with advanced cancer receiving palliative care: randomized controlled trial. Support Care Cancer 2017;25(9):2899-2908. doi: 10.1007/s00520-017$3742-4$

22. Husebø AML, Dyrstad SM, Mjaaland I, Søreide JA, Bru E. Effects of scheduled exercise on cancer-related fatigue in women with early breast cancer. Scientific World Journal 2014;19;2014:271828. doi: 10.1155/2014/271828

23. Samuel SR, Maiya AG, Fernandes DJ, Guddattu V, Prakash Saxena PU, Kurian JR, et al. Effectiveness of exercise-based rehabilitation on functional capacity and quality of life in head and neck cancer patients receiving chemo-radiotherapy. Support Care Cancer 2019;27(10):3913-20. doi: 10.1007/s00520-019-04750-z

24. Taaffe DR, Newton RU, Spry N, Joseph D, Chambers SK, Gardiner RA, et al. Effects of different exercise modalities on fatigue in prostate cancer patients undergoing androgen deprivation therapy: a year-long randomised controlled trial. Eur Urol 2017;72(2):29399. doi: 10.1016/j.eururo.2017.02.019

25. Baumann FT, Bieck O, Oberste M, Kuhn R, Schmitt J, Wentrock S, et al. Sustainable impact of an individualized exercise program on physical activity level and fatigue syndrome on breast cancer patients in two German rehabilitation centers. Supportive Care in Cancer 2016;25(4):1047-54. doi: 10.1007/s00520-016-3490-x

26. Schmidt ME, Wiskemann J, Armbrust P, Schneeweiss A, Ulrich CU, Steindorf K. Effects of resistance exercise on fatigue and quality of life in breast cancer patients undergoing adjuvant chemotherapy: a randomized controlled trial. Int J Cancer 2014;137(2):471-80. doi: 10.1002/ijc.29383

27. Grote M, Maihöfer C, Weigl M, Davies-Knorr P, Belka C. Progressive resistance training in cachectic head and neck cancer patients undergoing radiotherapy: a randomized controlled pilot feasibility trial. Radiat Oncol 2018;13(1):1-10. doi: 10.1186/s13014-0181157-0

28. Van Weert E, May AM, Korstjens I, Post WJ, van der Schans CP, et al. Cancer-related fatigue and rehabilitation: a randomized controlled multicenter trial comparing physical training combined with cognitive-behavioral therapy with physical training only and with no intervention. Phys Ther 2010;90(10):1413-25. doi: 10.2522/ptj.20090212

29. Andhare NM, Yeole UL, Varde F. Effect of physiotherapeutic exercises on fatigue and quality of life in cancer patients. Indian Journal of Public Health Research \& Development 2020;11(6):538-542. doi: 10.37506/ijphrd.v11i6.9834

30. Ammitzbøll G, Kjær TK, Johansen C, Lanng C, Andersen EW, Kroman N, et al. Effect of progressive resistance training on health-related quality of life in the first year after breast cancer surgery-results from a randomized controlled trial. Acta Oncol 2019;58(5):665-672. doi: 10.1080/0284186X.2018.1563718 
31. Rogers LQ, Vicari S, Trammell R, Hopkins-Price P, Fogleman A, Spenner A, et al. Biobehavioral factors mediate exercise effects on fatigue in breast cancer survivors. Med Sci Sports Exerc 2014;46(6):1077. doi: 10.1249/MSS.0000000000000210

32. Kampshoff CS, van Dongen JM, van Mechelen W, Schep G, Vreugdenhil A, Twisket JWR, et al. Long-term effectiveness and cost-effectiveness of high versus low-tomoderate intensity resistance and endurance exercise interventions among cancer survivors. J Cancer Surviv 2018;12(3):417-29. doi: 10.1007/s11764-018-0681-0 